\title{
Silibinin: A New Opportunity for the Treatment of Brain Metastasis from Lung Cancer
}

\author{
Raffaele Addeo (1D \\ U.O.C. Oncologia, "S. Giovanni di Dio" \\ Hospital, ASLNA2NORD, Naples, 80027, \\ Italy
}

\begin{abstract}
Despite multimodal treatment, the prognosis of brain metastases (BM) remains limited, with a survival of only a few months. In this report, silibinin (or silybin), a natural polyphenolic flavonoid isolated from seed extracts of the herb milk thistle, is discussed as a potential therapeutic option for the treatment of BM. This molecule has an anticancer effect, blocking the migratory and invasive properties of neoplastic cells. This mechanism is focused on controlling the signal transducer and activator of transcription 3 (STAT3)mediated pathway. STAT3 plays a major role in the growth of tumors and leads to metastasis, including BM. The promising but preliminary clinical results achieved by silibinin on lung cancer BM suggest new opportunities for combined treatment with radiotherapy and/or temozolomide, not just to limit severe neurological symptoms but also to control clinical progression of the disease.
\end{abstract}

Keywords: brain metastasis, lung cancer, silibinin, chemotherapy, target therapy, radiotherapy

Brain metastasis (BM) continues to represent a dramatic event that influences the prognosis of cancer patients, reducing their survival significantly. The involvement of systemic disease, the patient's performance status and age, and the number, location, size, and histology of the tumors may influence the prognosis and the therapeutic options. Recent treatment modalities include symptomatic care, such as antiepileptic drugs and corticosteroids, and definitive treatment. ${ }^{1}$ However, despite the incredible progress made in the treatment of tumors with new drugs such as immunotherapy, treatment choices are limited, and a small percentage of patients may benefit from surgery or radiosurgery. Whole brain radiotherapy (WBRT) represents an important choice for the majority of patients with BM, as it reduces symptoms and improves or stabilizes neurocognitive function; however, it is associated with poor survival, and temporary control of BM is achieved in half of the patients. However, long-term survivors have a high risk of developing neurocognitive disorders that impact their quality of life, and for this reason stereotactic radiosurgery is increasingly used in several countries.

Traditional chemotherapy has had a limited role thus far, while targeted therapies in specific molecular subgroups are increasingly being employed. ${ }^{2}$ The bloodbrain barrier, and incomplete knowledge of its properties and characteristics, represents one of the main obstacles. Therefore, it is crucial to increase the capacity of drugs to accumulate in brain tissue in adequate concentrations to induce antiproliferative effects. Certainly, promising data have emerged regarding
Correspondence: Raffaele Addeo U.O.C. Oncologia, "S. Giovanni di Dio" Hospital, ASLNA2NORD, Via Pirozzi Frattamaggiore, Naples, 80027, Italy Tel +390818891233

Fax +390818891353

Email raffaeleaddeol9@gmail.com;

raffaele.addeo@asInapoli2nord.it 
chemotherapeutic molecules such as temozolomide (TMZ), especially for primitive central nervous system tumors such as glioblastoma. ${ }^{3}$ Several clinical trials have been performed to investigate the association of TMZ and WBRT for the treatment of BM. ${ }^{4}$ Unfortunately, TMZ has proved to be completely ineffective against lung cancer BM. For this reason, it does not represent a gold standard in the clinical practice for the treatement of primary lung cancer.

Patients with BM who have oncogenic drivers, including $E G F R$ and $A L K$, may receive next-generation targeted therapy that is effective against central nervous system disease. In selected cases, immunotherapy may also achieve disease control for BM. ${ }^{5}$ Molecular target therapies, despite high expectations, did not provide clear improvement in the medical treatment of BM, with few exceptions. ${ }^{6}$ Nevertheless, the control of symptoms, together with the improvement in the quality of life, continues to represent the primary objective to study further.

Based on the discussion thus far, the recent scientific advances regarding silibinin (or silybin), and its efficacy against BM, deserve our attention and offer new stimuli for research and study. Silibinin inhibits the invasive capacities of metastatic cells, confirming that it possesses anticancer properties both in vivo and in vitro. ${ }^{7,8}$ This molecule has been shown to exert anticancer efficacy in well-known cancers, such as lung cancer, prostate cancer, colon cancer, breast cancer, bladder cancer, and hepatocellular carcinoma. ${ }^{9}$ Silybin, alone or in association with sorafenib, was shown to induce a cooperative antitumor action on the hepatocarcinoma cell line HepG-2, upregulating secretory tumor-suppressive miRNAs and downregulating secretory oncomiRNAs by secondary lipid metabolite production. ${ }^{10}$ A 2021 study confirmed the antineoplastic activity of silibinin, which inhibited the growth of lung adenocarcinoma in vitro and in vivo. Silibinin is a newly discovered inhibitor of TMEM16A, a novel lung cancer biomarker, which is closely related to the sustained proliferation of cancer cells. The authors showed that the inhibition of cancer growth is mediated by the phosphorylation of MEK1/2 and ERK1/2, and further reduces the expression of cyclin D1. ${ }^{11}$

This natural polyphenolic flavonoid inhibits multiple cancer cell signaling pathways, angiogenesis, regulation of epithelial-to-mesenchymal transition (EMT), adhesion, motility, and invasiveness. ${ }^{12}$

Silibinin inhibits signal transducer and activator of transcription 3 (pSTAT3) in several cancer models, such as prostate, breast, skin, and lung. ${ }^{13,14}$ Several reports (up to 2007) have reported the role of this molecule in reducing the constitutive STAT3 phosphorylation. ${ }^{15}$

The dysregulation and malfunction of STAT3 contribute to cancer initiation and progression, and are involved in the tumor/metastasis-initiating capacity and resistance to therapy. ${ }^{16}$ STAT3 has been shown to be important in $\mathrm{BM}$, and it plays a central role in the control of a few molecules in the microenvironment, such as $\mathrm{CD}^{+} \mathrm{T}$ cells, reactive astrocytes, and microglia, during metastatic colonization of the brain. A genetic approach, targeting STAT3 specifically in reactive astrocytes, was able to reproduce the benefit obtained with silibinin, which suggests that the main role of silibinin is derived from the protumorigenic role of this subpopulation of the microenvironment. ${ }^{17}$

In contrast to first-line treatment, the treatment for recurrent or progressive BM is not unanimously accepted and shared, especially for symptomatic patients with poor performance status, for whom only supportive and symptomatic care is preferred to preserve their quality of life. The use of supportive therapy with supplements for cancer patients is common, although these therapeutic options are not recommended by oncologists, who do not consider them useful for the improvement of tumor symptoms. ${ }^{18}$ A study investigating the benefits of the silibinin-based nutraceutical Legasil ${ }^{\circledR}$ resulted in significant improvement in patients with nonsmall cell lung cancer after WBRT and chemotherapy and who had presented poor performance status. ${ }^{19}$ The use of this therapeutic option for the treatment of BM is also corroborated by evidence of a documented antiedema activity of silibinin. In an experimental mouse model of ischemic stroke, the administration of silibinin achieved a significant improvement in neurological symptoms, with a reduction in edema and improvement in motor functions. ${ }^{20}$ These properties of silibinin open up new scenarios for combined treatment with radiotherapy, not only with the aim of controlling the severe neurological symptoms but also with the more ambitious aim of controlling the progression of the disease. Priego et al achieved promising results with Legasil in heavily pretreated patients with BM, confirming the benefits of this therapy in patients with BM. ${ }^{21}$ The investigators demonstrated a clear improvement in overall survival in the group of BM patients treated with Legasil (18 patients) versus the control cohort: 15.5 months versus 4 months, respectively. In these experiments, the authors used a specific formulation of silibinin (Eurosil85) as part of the nutraceutical Legasil, created to improve its very poor bioavailability. This was predicted to make significant differences to their transport 
and blood-brain barrier permeability compared with other formulations derived from silibinin. This formulation may account for the effective activity of such a specific formulation of silibinin against BM, as it crossed the blood-brain barrier. $^{22}$ The favorable toxicity profile and few side effects make it relatively easy and desirable to further study these ambitious but preliminary results through randomized studies aimed at better understanding the role of silibinin, both alone and in association with radiotherapy or other anticancer drugs that are effective against lung cancer, for the treatment of lung cancer BM.

\section{Disclosure}

The author reports no conflicts of interest in this work.

\section{References}

1. Moravan MJ, Fecci PE, Anders CK, et al. Current multidisciplinary management of brain metastases. Cancer. 2020;126(7):1390-1406. doi: $10.1002 /$ cncr.32714

2. Soffietti R, Ahluwalia M, Lin N, Rudà R. Management of brain metastases according to molecular subtypes. Nat Rev Neurol. 2020;16(10):557-574. doi:10.1038/s41582-020-0391-x

3. Feng E, Sui C, Wang T, Sun G. Temozolomide with or without radiotherapy in patients with newly diagnosed glioblastoma multiforme: a meta-analysis. Eur Neurol. 2017;77(3-4):201-210. doi:10.1159/ 000455842

4. Addeo R, Caraglia M. Combining temozolomide with other antitumor drugs and target-based agents in the treatment of brain metastases: an unending quest or chasing a chimera? Expert Opin Investig Drugs. 2011;20(7):881-895. doi:10.1517/13543784.2011.580736

5. Bulbul A, Forde PM, Murtuza A, et al. Systemic treatment options for brain metastases from non-small-cell lung cancer. Oncol Williston Park. 2018;32(4):156-163.

6. Caraglia M, Addeo R. target therapy in brain tumours and metastases. Curr Cancer Drug Targets. 2012;3:18. doi:10.2174/1568009 12799277458

7. Deep G, Agarwal R. Antimetastatic efficacy of silibinin: molecular mechanisms and therapeutic potential against cancer. Cancer Metastasis Rev. 2010;29(3):447-463. doi:10.1007/s10555-010-9237-0

8. Jahanafrooz Z, Motamed N, Rinner B, Mokhtarzadeh A, Baradaran B. Silibinin to improve cancer therapeutic, as an apoptotic inducer, autophagy modulator, cell cycle inhibitor, and microRNAs regulator. Life Sci. 2018;213:236-247. doi:10.1016/j.lfs.2018.10.009
9. Zhu XX, Ding YH, Wu Y, et al. Silibinin: a potential old drug for cancer therapy. Expert Rev Clin Pharmacol. 2016;9(10):1323-1330. doi:10.1080/17512433.2016.1208563

10. Zappavigna S, Vanacore D, Lama S, et al. Silybin-induced apoptosis occurs in parallel to the increase of ceramides synthesis and miRNAs secretion in human hepatocarcinoma cells. Int J Mol Sci. 2019;20 (9):2190. doi:10.3390/ijms20092190

11. Guo S, Bai X, Liu Y, et al. Inhibition of TMEM16A by natural product silibinin: potential lead compounds for treatment of lung adenocarcinoma. Front Pharmacol. 2021;12:643489. doi:10.3389/ fphar.2021.643489

12. Ramasamy K, Agarwal R. Multitargeted therapy of cancer by silymarin. Cancer Lett. 2008;269(2):352-362. doi:10.1016/j. canlet.2008.03.053

13. Binienda A, Ziolkowska S. the anticancer properties of silibinin: its molecular mechanism and therapeutic effect in breast cancer. Anticancer Agents Med Chem. 2020;20(15):1787-1796. doi:10.2174/1871520620666191220142741

14. Prasad RR, Paudel S, Raina K, Agarwal R. Silibinin and non-melanoma skin cancers. J Tradit Complement Med. 2020;10 (3):236-244. doi:10.1016/j.jtcme.2020.02.003

15. Bosch-Barrera J, Menendez JA. Silibinin and STAT3: a natural way of targeting transcription factors for cancer therapy. Cancer Treat Rev. 2015;41(6):540-546. doi:10.1016/j.ctrv.2015.04.008

16. Wu M, Song D, Li H, et al. Negative regulators ofSTAT3signaling pathway in cancers. Cancer Manag Res. 2019;11:4957-4969. doi:10.2147/CMAR.S206175

17. Li L, Zeng J, Gao Y, He D. Targeting silibinin in the antiproliferative pathway. Expert Opin Investig Drugs. 2010;19(2):243-255. doi:10.1517/13543780903533631

18. Frenkel M. Is there a role for homeopathy in cancer care? Questions and challenges. Curr Oncol Rep. 2015;17(9):43. doi:10.1007/s11912015-0467-8

19. Bosch-Barrera J, Sais E, Cañete N, et al. Response of brain metastasis from lung cancer patients to an oral nutraceutical product containing silibinin. Oncotarget. 2016;7(22):32006-32014. doi:10.18 632/oncotarget. 7900

20. Wang C, Wang Z, Zhang $X$, et al. Protection by silibinin against experimental ischemic stroke: up-regulated pAkt, pmTOR, HIF-1 $\alpha$ and Bcl-2, down-regulated Bax, NF- $\mathrm{KB}$ expression. Neurosci Lett. 2012;529(1):45-50. doi:10.1016/j.neulet.2012.08.078

21. Priego N, Zhu L, Monteiro C, et al. STAT3 labels a subpopulation of reactive astrocytes required for brain metastasis. Nat Med. 2018;24 (7):1024-1035. doi:10.1038/s41591-018-0044-4

22. Pérez-Sánchez A, Cuyàs E, Ruiz-Torres V, et al. Intestinal permeability study of clinically relevant formulations of silibinin in Caco-2 cell monolayers. Int J Mol Sci. 2019;20(7):1606. doi:10.3390/ ijms20071606
The Journal of Experimental Pharmacology is an international, peerreviewed, open access journal publishing original research, reports, reviews and commentaries on all areas of laboratory and experimental pharmacology. The manuscript management system is completely online and includes a very quick and fair peer-review system. Visit http://www.dovepress.com/testimonials.php to read real quotes from published authors. 